\title{
Realization of Negative Group Delay Network Using Defected Microstrip Structure
}

\author{
Girdhari Chaudhary, ${ }^{1}$ Yongchae Jeong, ${ }^{1}$ and Jongsik Lim $^{2}$ \\ ${ }^{1}$ Division of Electronics Engineering, IT Convergence Research Center, Chonbuk National University, \\ Jeollabuk-do, Jeonju 561-756, Republic of Korea \\ ${ }^{2}$ Department of Electrical Engineering, Soonchunhyang University, Chungnam, Asan 336-745, Republic of Korea
}

Correspondence should be addressed to Yongchae Jeong; ycjeong@jbnu.ac.kr

Received 23 December 2013; Accepted 25 March 2014; Published 8 April 2014

Academic Editor: Hon Tat Hui

Copyright (C) 2014 Girdhari Chaudhary et al. This is an open access article distributed under the Creative Commons Attribution License, which permits unrestricted use, distribution, and reproduction in any medium, provided the original work is properly cited.

A design of negative group delay (NGD) networks using a U-shaped defected microstrip structure (DMS) and lumped elements is presented in this paper. The signal attenuation characteristics of DMS were utilized to get NGD time. The group delay (GD) time and signal attenuation of the proposed networks are controlled by an external resistor connected across the DMS slot. For experimental validation, a single-stage and cascaded two-stage NGD networks were designed and fabricated. From experimental results, the GD of $-8.24 \pm 1.1$ ns with the maximum insertion loss of $37.84 \mathrm{~dB}$ was obtained over bandwidth of $40 \mathrm{MHz}$.

\section{Introduction}

In recent years, there has been an increasing amount of research on negative group delay (NGD) networks. The NGD is a counterintuitive phenomenon that relates time advancement to wave propagation [1-12]. This NGD phenomenon can be observed within the limited frequency band of signal in certain media under signal attenuation condition. It has been implemented in electronic circuitry and applied to various practical applications in communication systems, such as shortening or reducing delay lines, efficiency enhancement of a feedforward linear amplifier, bandwidth enhancement of a feedback linear amplifier, and beam-squint minimization in phased array antenna systems $[3-6,11]$. The group delay (GD) characteristics in circuit can be investigated by examining phase variation of forward transmitting scattering parameter. Using the deferential-phase GD $\left(\tau_{g}\right)$ relation,

$$
\tau_{g}=-\frac{d \varphi}{d \omega},
$$

the presence of NGD in circuit is equivalent to an increasing phase (positive phase slope) with frequencies.
The various kinds of NGD network based on active/ passive RLC resonators have been theoretically and experimentally validated in the previous studies [2-12]. However, none of previous works were implemented transmission-type configuration NGD network using a parallel RLC resonator with a distributed transmission line because of implementation difficulty.

Recently, there is a growing interest on the periodic structures such as microstrip photonic bandgap (PBG), defected ground structure (DGS), and defected microstrip structure (DMS) which provide the signal attenuation characteristic at certain resonant frequency and are applied successfully in various applications $[2,8]$. The DMS is a patterned structure etched on the signal strip instead of the ground plane. However, none of previous works were focused to design NGD network using DMS.

In this paper, the attenuation characteristics of DMS are utilized to design and investigate NGD networks. First, the U-shaped DMS is investigated, and the equivalent lumped elements are extracted using the equivalent circuit model. Secondly, the external capacitor is connected across the DMS slot to get the required operating resonant frequency. Then, 


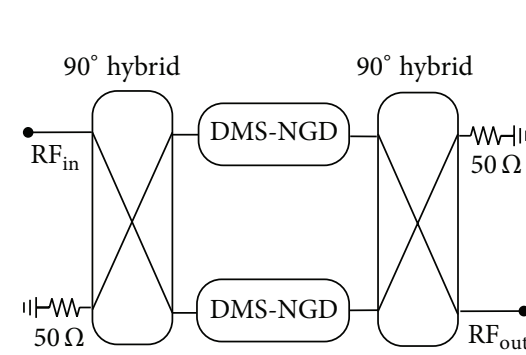

(a)

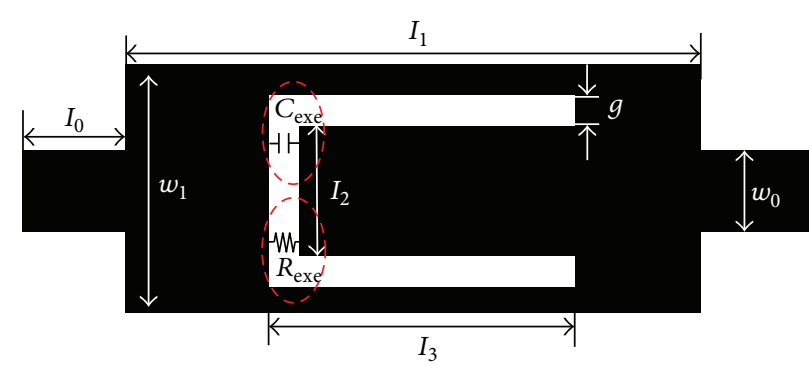

(b)

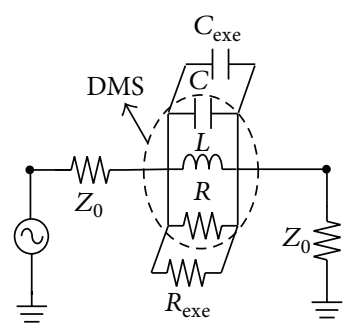

(c)

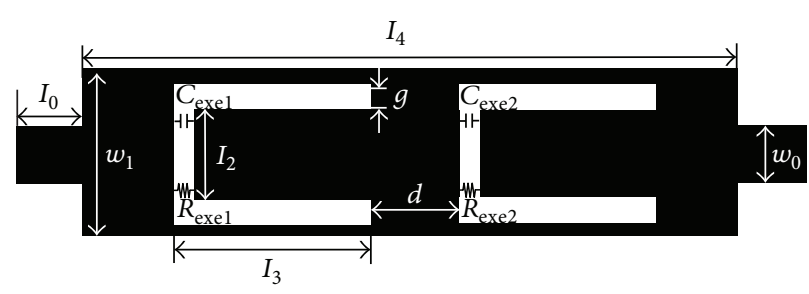

(d)

FIGURE 1: Proposed NGD networks: (a) configuration of balanced-type structure, (b) single-stage DMS-NGD network, (c) equivalent circuit of a single-stage DMS-NGD network, and (d) cascaded two-stage network.

the required amount of NGD time at the operating frequency is obtained by connecting the external resistor.

\section{Design and Implementation}

The configuration of the proposed NGD network is shown in Figure 1(a) which consists of two $90^{\circ}$ hybrid couplers and DMS-NGD networks. The balanced-type hybrid structure aims to improve the return loss characteristics of the transmission-type configuration NGD network. The layout of the DMS-NGD network is shown in Figure 1(b) consisting of an external lumped capacitor $\left(C_{\text {exe }}\right)$ and a resistor $\left(R_{\text {exe }}\right)$ connected across the DMS slot. The equivalent circuit model of DMS can be expressed as a parallel RLC circuit as shown in Figure 1(c) and the equivalent lumped elements of DMS are extracted by performing an EM simulation [13] which are given as (2a), (2b), and (2c)

$$
\begin{gathered}
C=\frac{f_{c}}{4 \pi Z_{0}\left(f_{0}^{2}-f_{c}^{2}\right)}, \\
L=\frac{1}{4 \pi^{2} f_{0}^{2} C}, \\
R=2 Z_{0} \frac{1-S_{21}\left(f_{0}\right)}{S_{21}\left(f_{0}\right)},
\end{gathered}
$$

where $f_{c}, f_{0}$, and $S_{21}$ are $3 \mathrm{~dB}$ cut-off frequency, resonant frequency, and transmission coefficient at the resonant frequency obtained from EM simulation, respectively.

The externally connected $C_{\text {exe }}$ and $R_{\text {exe }}$ are in parallel with the RLC equivalent circuit of DMS as shown in Figure 1(c). The added $C_{\text {exe }}$ is used to obtain the required resonant frequency and GD time, whereas $R_{\text {exe }}$ is used to get the required value of transmission characteristics at the resonant frequency. From the equivalent circuit of proposed structure shown in Figure 1(c), the GD and signal attenuation $\left(S_{21}\right)$ at the resonant frequency can be calculated as (3a) and (3b)

$$
\begin{gathered}
\left.\tau\right|_{f=f_{0}}=-\frac{1}{2 \pi} \frac{d \angle S_{21}}{d f}=-\frac{2 R_{t}^{2} C_{t}}{2 Z_{0}+R_{t}} \\
\left|S_{21}\right|_{f=f_{0}}=\frac{2 Z_{0}}{2 Z_{0}+R_{t}},
\end{gathered}
$$

where $Z_{0}$ is termination port impedance and the values of $C_{t}$ and $R_{t}$ are given as $(4 \mathrm{a})$ and $(4 \mathrm{~b})$

$$
\begin{aligned}
& C_{t}=C+C_{\text {exe }}, \\
& R_{t}=\frac{R R_{\text {exe }}}{R+R_{\text {exe }}} .
\end{aligned}
$$

From (3a) and (4a) and (4b), it is clear that the GD time is controlled by $R_{\text {exe }}$ and $C_{t}$. To verify the design concept of the proposed NGD network, firstly, the U-shaped DMS is simulated with a full-wave solver Ansoft HFSSv13 with the following dimensions: $w_{0}=2.4, l_{0}=2, w_{1}=4, l_{1}=16, l_{2}=6.8$, $l_{3}=2.8$, and $g=0.4$ (all units are in $\mathrm{mm}$ ). The simulation is performed using a substrate RT/duroid 5880 with dielectric constant $\left(\varepsilon_{r}\right)$ of 2.2 and thickness $(h)$ of 31 mils.

Figure 2 shows the simulated resonance characteristics of $\mathrm{U}$-shaped DMS where $3 \mathrm{~dB}$ cut-off and resonant frequencies are at $5.66 \mathrm{GHz}$ and $7.56 \mathrm{GHz}$. Therefore, the extracted values of equivalent circuit of DMS are given as $C=0.3586 \mathrm{pF}$, $L=1.2389 \mathrm{nH}$, and $R=3.5119 \mathrm{k} \Omega$, respectively. Since the capacitance of DMS only is not enough to get the required resonant frequency of $2.14 \mathrm{GHz}$; therefore, by connecting $C_{\text {exe }}=4.15 \mathrm{pF}$, the resonant frequency is moved toward lower frequency around $2.14 \mathrm{GHz}$ as shown in Figure 2. Similarly, 


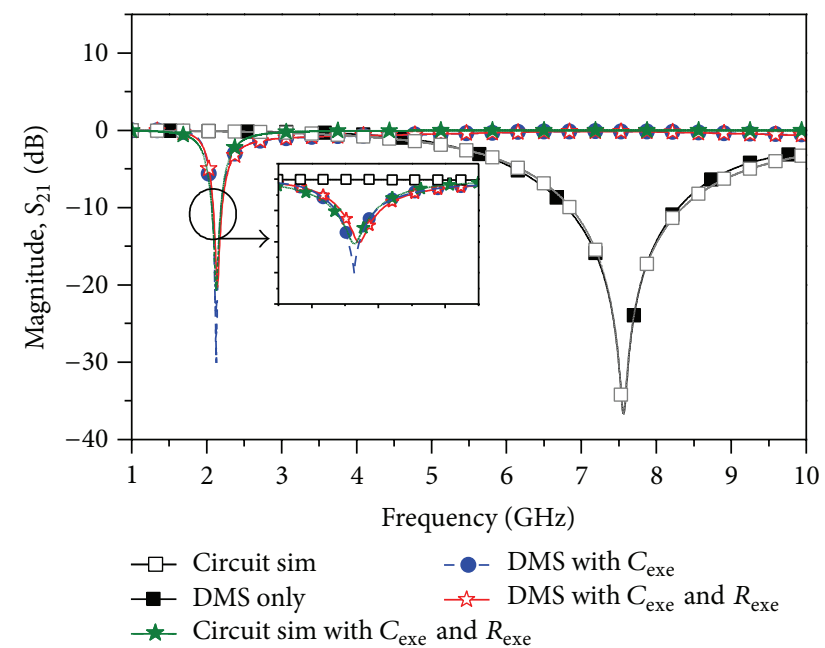

FIGURE 2: Full-wave and circuit simulated transmission response of U-shaped DMS with external lumped elements.

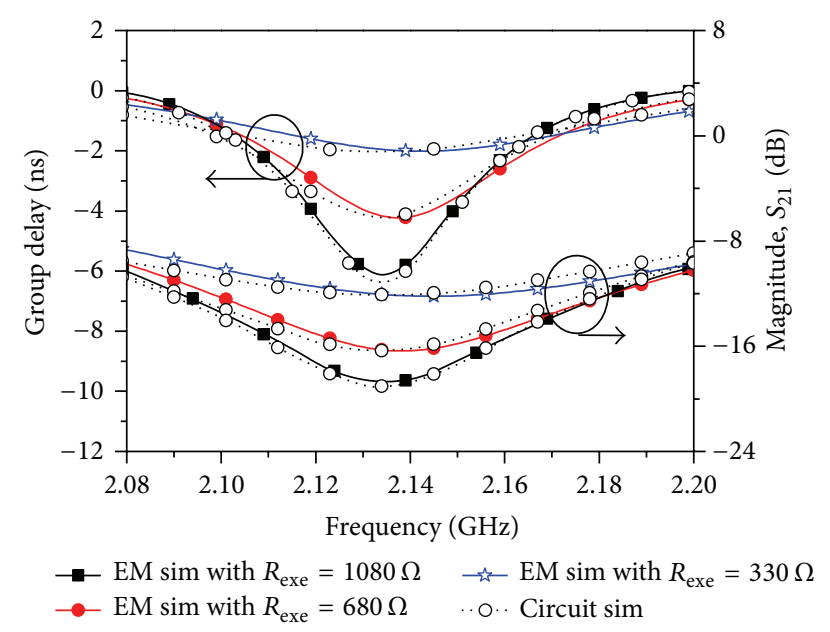

FIGURE 3: Simulated group delay and insertion loss characteristics of the proposed NGD network.

the transmission coefficient $\left(S_{21}\right)$ is controlled by connecting $R_{\text {exe }}=1.1 \mathrm{k} \Omega$.

Figure 3 shows the simulation results of NGD network under different $R_{\text {exe }}$. The circuit simulation results have a good agreement with EM-simulation results. From this figure, it is shown that the GD amount is controlled by $R_{\text {exe }}$. From the simulation, the GD of $-6.5 \mathrm{~ns}$ with insertion loss $19.4 \mathrm{~dB}$ was obtained at center frequency $2.14 \mathrm{GHz}$.

\section{Simulation and Measurement Results}

The goal was to design the GD of $-7 \mathrm{~ns}$ for the wideband code division multiple access (WCDMA) downlink band operating at the center frequency $2.14 \mathrm{GHz}$. For this purpose, the NGD network using DMS is simulated and fabricated. The physical dimensions of U-shaped DMS are same as previous.
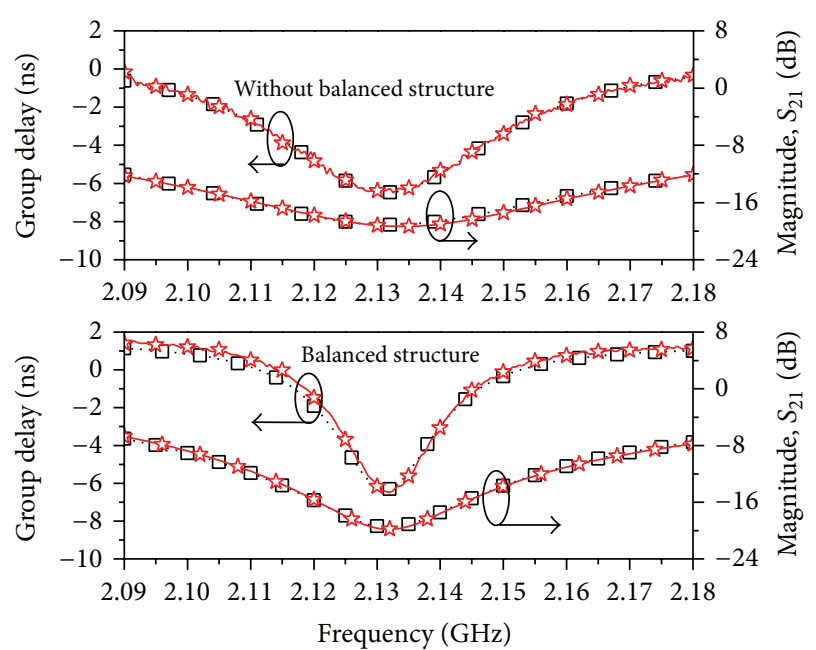

ㅁ. . Sim

$\rightarrow-$ Meas

(a)

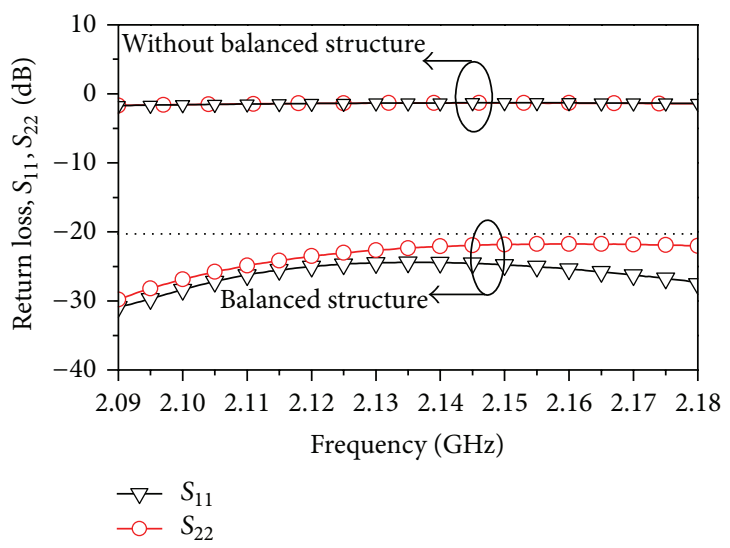

(b)

FIGURE 4: Single-stage NGD networks: (a) simulated and measured group delay and magnitude characteristics and (b) measured return loss characteristics.

Figure 4(a) shows the simulation and measurement results of single-stage NGD networks (with and without balanced structure). The measurement results have a good agreement with the EM-simulation results. In this measurement, $C_{\text {exe }}=4.14 \mathrm{pF}$ and $R_{\text {exe }}=1.1 \mathrm{k} \Omega$ are used. From the measurement, it is found that the GD of -6.53 ns with the maximum signal attenuation of $19.97 \mathrm{~dB}$ at the center frequency of $2.14 \mathrm{GHz}$ in case of balanced type.

The comparison results of return loss characteristics are shown in Figure 4(b). As seen from this figure, return loss characteristics are around $1.24 \mathrm{~dB}$ in case of without balanced structure. Therefore, the balanced-type structure was used in order to improve the return loss $\left(S_{11}\right.$ and $\left.S_{22}\right)$ characteristics. The input and output return loss $\left(S_{11}\right.$ and $\left.S_{22}\right)$ characteristics of balanced- type NGD network are better than $23.5 \mathrm{~dB}$.

As seen from Figure 4(a), the NGD bandwidth is small which prevents to be used in practical applications. Therefore, the NGD bandwidth enhancement is required. For 


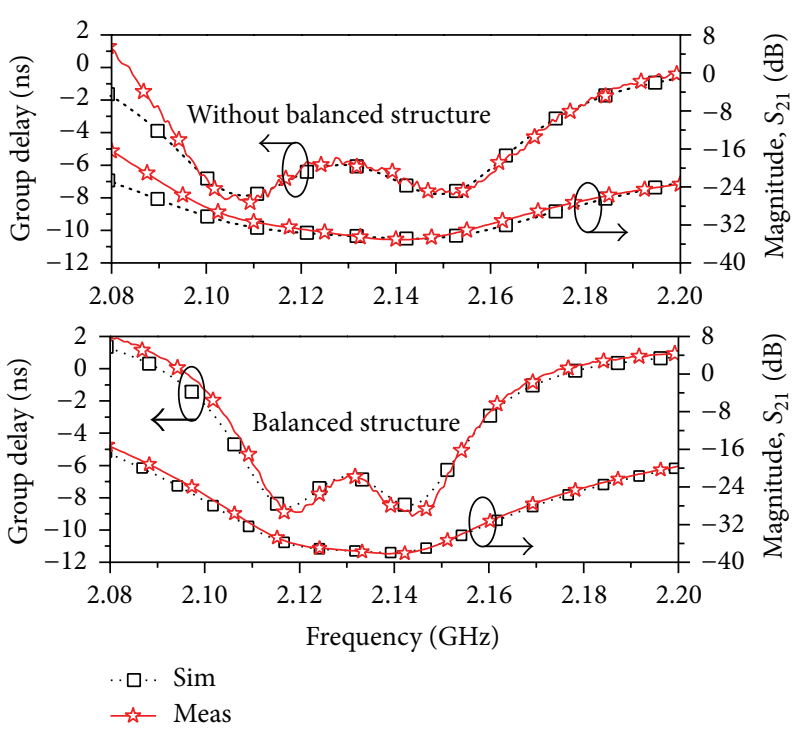

(a)

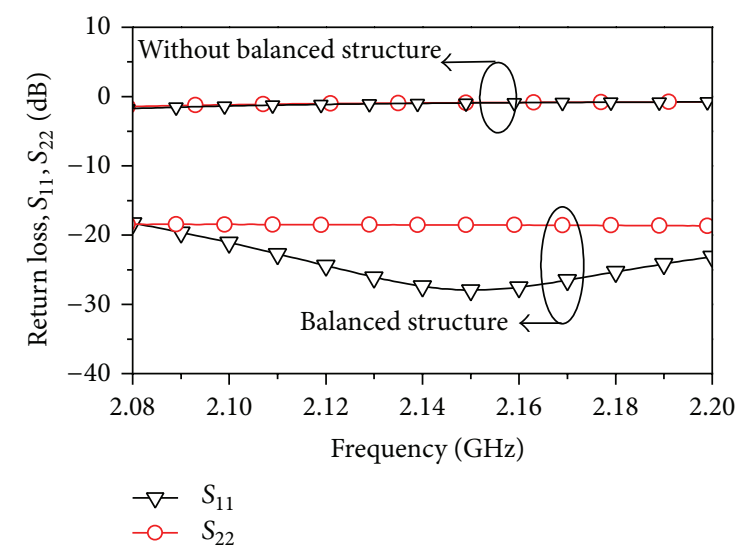

(b)

FIGURE 5: Two-stage DMS-NGD networks: (a) simulated and measured group delay and magnitude characteristics and (b) measured return loss characteristics.

this purpose, the two-unit NGD network cells operating at center frequencies of $2.10 \mathrm{GHz}$ and $2.15 \mathrm{GHz}$, respectively, are cascaded as shown in Figure 1(d). The physical dimensions of the unit cell DMS-NGD network are the same as described previously, except for the separation distance between the unit cells, which is given as $5.4 \mathrm{~mm}$. The values of $C_{\text {exel }}=$ $4.16 \mathrm{pF}, R_{\text {exe1 }}=1.1 \mathrm{k} \Omega, C_{\text {exe2 }}=4.10 \mathrm{pF}$, and $R_{\text {exe2 }}=1.1 \mathrm{k} \Omega$ are used to achieve the desired operating center frequency and GD time, respectively.

Figure 5(a) shows the simulation and measurement results of balanced-type two-stage NGD networks. The measurement results are in good agreement with the simulation results. From the measurements, it was found that the GD time was $-8.24 \pm 1.1 \mathrm{~ns}$ over an operating bandwidth of $40 \mathrm{MHz}$ in case of balanced-type. The measured maximum signal attenuation at $2.125 \mathrm{GHz}$ was around $37.84 \mathrm{~dB}$. As seen from Figures 4(a) and 5(a), the NGD bandwidth of balanced-type NGD networks have slightly decreased as
TABLE 1: Performance comparison of proposed NGD networks with conventional.

\begin{tabular}{lcccccc}
\hline & $\begin{array}{c}f_{o} \\
(\mathrm{GHz})\end{array}$ & $\begin{array}{c}\mathrm{GD}_{\max } \\
(\mathrm{ns})\end{array}$ & $\begin{array}{c}\mathrm{S}_{21 \max } \\
(\mathrm{dB})\end{array}$ & $\begin{array}{c}\text { NGD } \\
\mathrm{BW} \\
(\mathrm{MHz})\end{array}$ & $\begin{array}{c}\text { NGD } \\
\mathrm{BW} \\
\text { product }\end{array}$ & $\begin{array}{c}\text { Return } \\
\text { loss } \\
(\mathrm{dB})\end{array}$ \\
\hline$[7]^{*}$ & 1.00 & -2.30 & 1.680 & $\mathrm{X}$ & $\mathrm{X}$ & -10 \\
{$[9]^{*}$} & 0.310 & -1.52 & 0.290 & 120 & 0.182 & -15 \\
{$[10]^{*}$} & 0.454 & -1.52 & 0.300 & 103 & 0.156 & -15 \\
{$[3,4,11]$} & 2.140 & -9.00 & -62.40 & 30 & 0.270 & $<-20$ \\
{$[12]$} & 4.500 & -0.05 & -10.00 & 3000 & 0.150 & $\mathrm{X}$ \\
{$[2]$} & 3.505 & -3.80 & -37.16 & 80 & 0.304 & $\mathrm{X}$ \\
This & $\mathbf{2 . 1 2 5}$ & $-\mathbf{8 . 2 0}$ & $-\mathbf{3 7 . 8 4}$ & $\mathbf{4 0}$ & $\mathbf{0 . 3 2 8}$ & $<-\mathbf{1 9}$ \\
work & & & & & &
\end{tabular}

${ }^{*}$ Active NGD network.
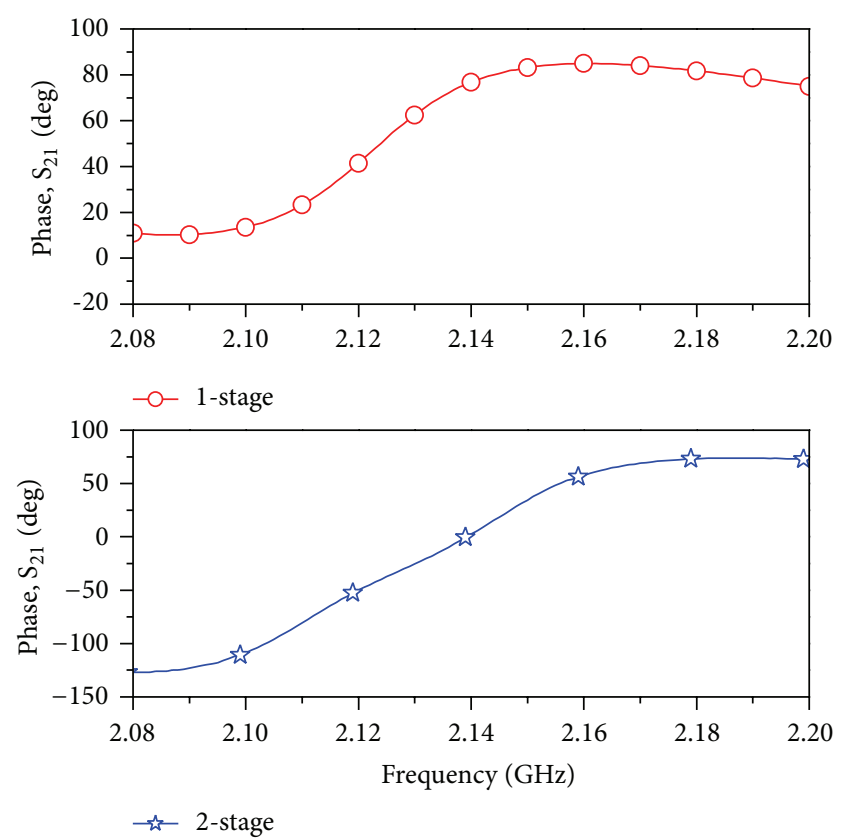

FIGURE 6: Measured phase characteristics of balanced type NGD networks.

compared to without balanced structure because it should be compensated positive group delay of two $90^{\circ}$ hybrid couplers by designing NGD networks for higher value of NGD time than actual required value. As NGD time is increased, the NGD bandwidth decrease as well as increase the signal attenuation. The return loss characteristics of cascaded twostage NGD networks are shown in Figure 5(b). The return loss characteristics are around $1 \mathrm{~dB}$ in case of without balanced structure whereas these characteristics are better than $19 \mathrm{~dB}$ in case of balanced type.

Figure 6 shows the measured phase characteristics of single and cascaded two-stage balanced type NGD networks. As seen in the figures, the slope of the transmission phase is positive in a certain region of the frequency, which signifies the presence of the NGD in the proposed circuit. The signal 


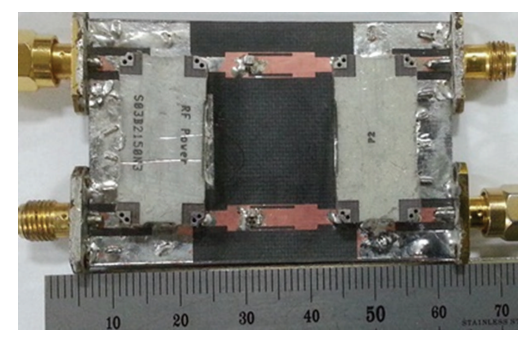

(a)

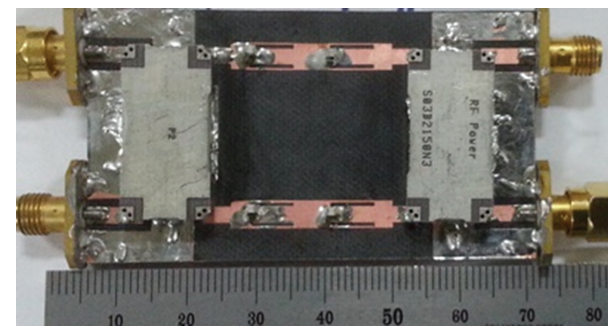

(b)

Figure 7: Photographs of fabricated circuits: (a) single-stage and (b) cascaded two-stage circuits.

attenuations can be compensated by using general purpose gain amplifiers. The photographs of fabricated circuit are shown in Figure 7. The performance comparison of the proposed network with conventional circuits is given in Table 1. As seen from this table, the proposed NGD network provides the highest NGD-bandwidth among conventional circuits.

\section{Conclusion}

In this paper, the demonstration of planar balanced-type negative group delay networks using a defected microstrip structure and lumped elements is presented. By taking advantage of the attenuation characteristics of the defected microstrip structure and connecting an external resistor and capacitor, the desired negative group delay and operating center frequency were obtained. The negative group delay is independently controlled by an external resistor. The proposed negative group delay network has compact size that is easy to implement. Therefore, it is expected to be applicable in communication systems such as minimizing the antenna correlation between adjacent antennas in multipleinput-multiple-output (MIMO) systems. It could also be applied in realization of non-Foster elements as well as electromagnetics application such as enhancing bandwidth of artificial magnetic conductors (AMCs).

\section{Conflict of Interests}

The authors declare that there is no conflict of interests regarding the publication of this paper.

\section{Acknowledgments}

This work was supported by the Basic Science Research Program through the National Research Foundation of Korea (NRF) funded by the Ministry of Education, Science and Technology (2013006660).

\section{References}

[1] M. Kitano, T. Nakanishi, and K. Sugiyama, "Negative group delay and superluminal propagation: an electronic circuit approach," IEEE Journal on Selected Topics in Quantum Electronics, vol. 9, no. 1, pp. 43-51, 2003.

[2] G. Chaudhary, J. Jeong, P. Kim, Y. Jeong, and J. Lim, "Compact negative group delay circuit using defected ground structure," in Proceedings of the IEEE Asia-Pacific Microwave Conference, pp. 22-24, November 2013.

[3] H. Choi, Y. Jeong, C. D. Kim, and J. S. Kenney, "Efficiency enhancement of feedforward amplifiers by employing a negative group-delay circuit," IEEE Transactions on Microwave Theory and Techniques, vol. 58, no. 5, pp. 1116-1125, 2010.

[4] Y. Jeong, H. Choi, and C. D. Kim, "Experimental verification for time advancement of negative group delay in RF electronic circuits," Electronics Letters, vol. 46, no. 4, pp. 306-307, 2010.

[5] S.-S. Oh and L. Shafai, "Compensated circuit with characteristics of lossless double negative materials and its application to array antennas," IET Microwaves, Antennas and Propagation, vol. 1, no. 1, pp. 29-38, 2007.

[6] B. Ravelo, M. le Roy, and A. Pérennec, "Application of negative group delay active circuits to the design of broadband and constant phase shifters," Microwave and Optical Technology Letters, vol. 50, no. 12, pp. 3078-3080, 2008.

[7] B. Ravelo, A. Pérennec, M. L. Roy, and Y. G. Boucher, "Active microwave circuit with negative group delay," IEEE Microwave and Wireless Components Letters, vol. 17, no. 12, pp. 861-863, 2007.

[8] D. Ahn, J.-S. Park, C.-S. Kim, J. Kim, Y. Qian, and T. Itoh, "A design of the low-pass filter using the novel microstrip defected ground structure," IEEE Transactions on Microwave Theory and Techniques, vol. 49, no. 1, pp. 86-93, 2001.

[9] M. Kandic and G. E. Bridges, "Bilateral gain-compensated negative group delay circuit," IEEE Microwave and Wireless Components Letters, vol. 21, no. 6, pp. 308-310, 2011.

[10] M. Kandic and G. E. Bridges, "Asymptotic limits of negative group delay in active resonator-based distributed circuits," IEEE Transactions on Circuits and Systems I: Regular Papers, vol. 58, no. 8, pp. 1727-1735, 2011.

[11] H. Choi, Y. Jeong, C. D. Kim, and J. S. Kenney, "Bandwidth enhancement of an analog feedback amplifier by employing a negative group delay circuit," Progress in Electromagnetics Research, vol. 105, pp. 253-272, 2010.

[12] C. D. Broomfield and J. K. A. Everard, "Broadband negative group delay networks for compensation of microwave oscillators and filters," Electronics Letters, vol. 36, no. 23, pp. 1931-1933, 2000.

[13] J. A. Tirado-Méndez, H. Jardón-Aguilar, F. Iturbide-Sánchez, I. Garcia-Ruiz, V. Molina-Lopez, and R. Acevo-Herrera, "A proposed defected microstrip structure (DMS) behavior for reducing rectangular patch antenna size," Microwave and Optical Technology Letters, vol. 43, no. 6, pp. 481-484, 2004. 

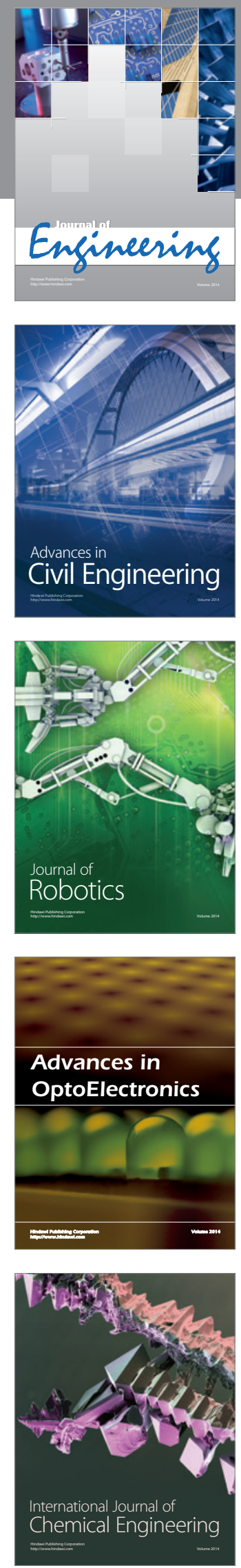

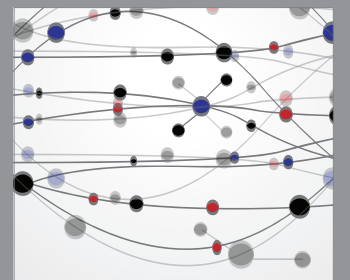

The Scientific World Journal
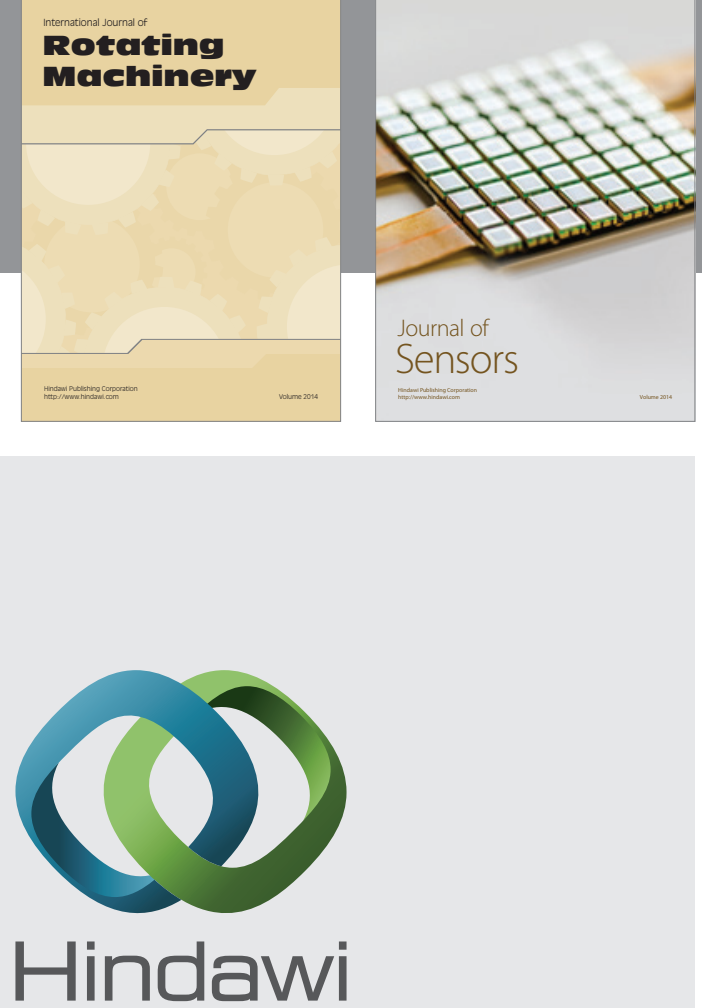

Submit your manuscripts at http://www.hindawi.com
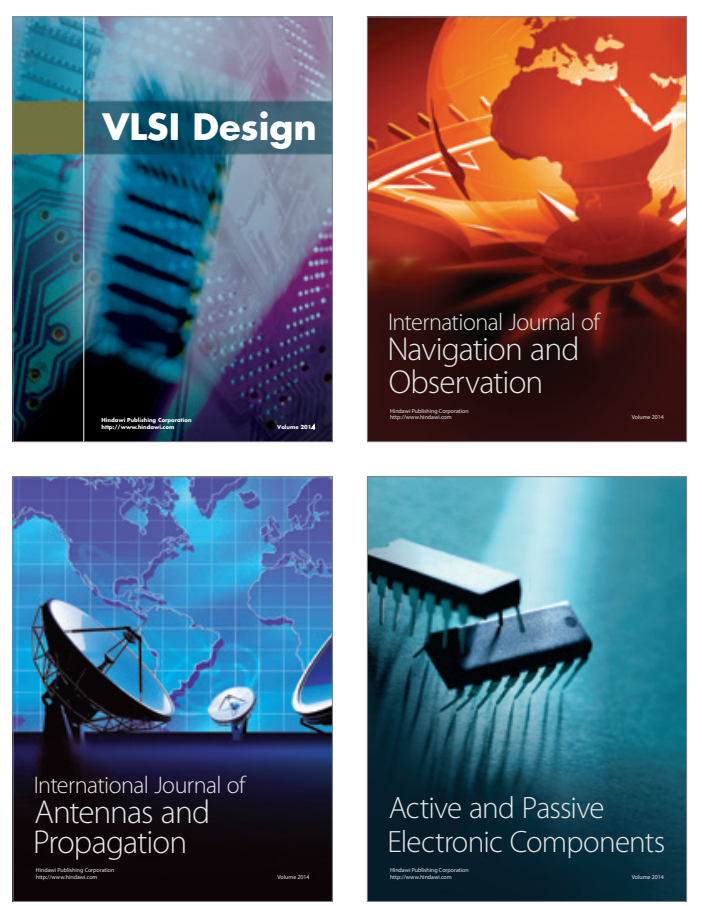
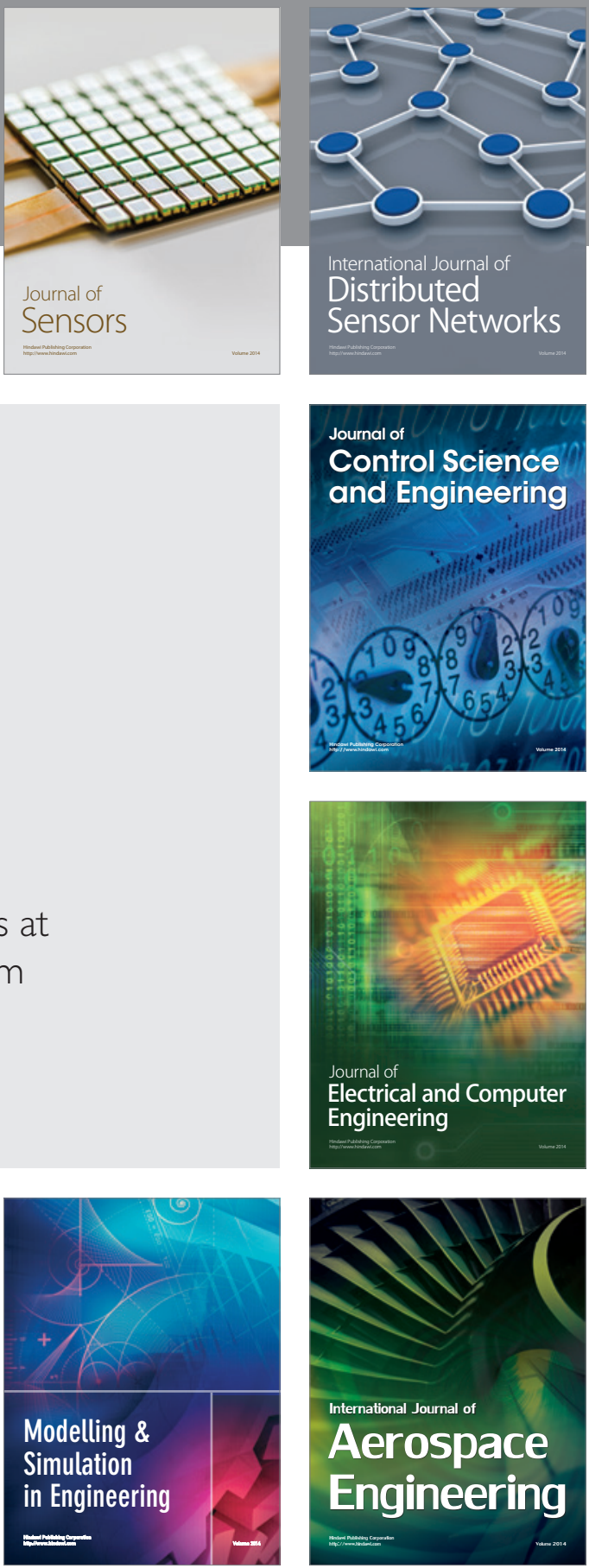

Journal of

Control Science

and Engineering
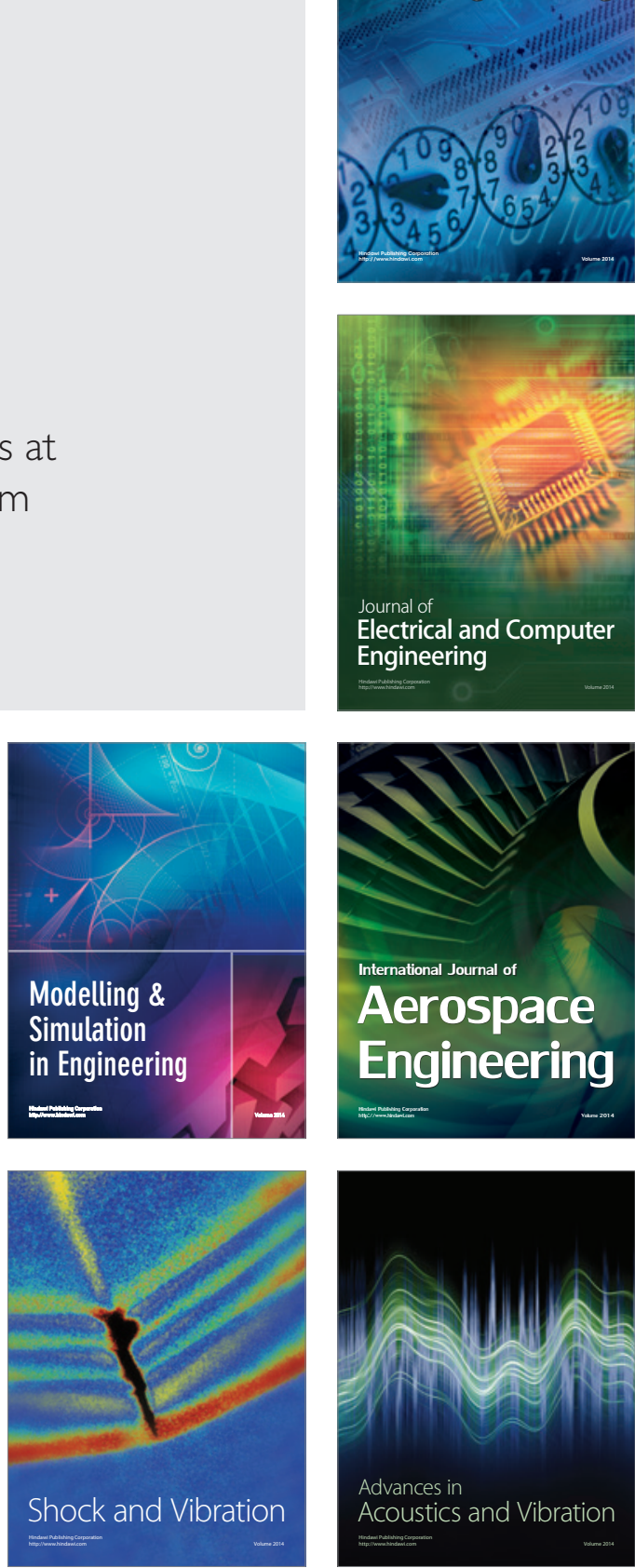